\title{
LA CONTRIBUCIÓN DE LA ERGONOMÍA AL PROCESO DE REGRESO AL TRABAJO Y LOS RETOS DE INCLUIR PROFESIONALES DE ENFERMERÍA: LA EXPERIENCIA DE UN HOSPITAL EN BRASIL
}

\author{
THE CONTRIBUTION OF ERGONOMICS TO THE RETURN TO WORK PROCESS AND \\ THE CHALLENGES OF INCLUDING NURSING PROFESSIONALS: THE EXPERIENCE OF \\ A HOSPITAL IN BRAZIL
}

\author{
Angélica García-Juns* \\ Octávio Augusto Camilo de Oliveira* ${ }^{* *}$ \\ Gabriela Moutinho-Florez ${ }^{* * *}$
}

\begin{abstract}
Resumen: Este artículo es un relato de la experiencia como ergónoma en un hospital privado brasileño, en prácticas de apoyo a los profesionales de enfermería para que regresen al trabajo luego de períodos prolongados de baja médica. El desarrollo institucional de un Programa de Retorno al Trabajo involucra a un equipo que busca garantizar el apoyo y garantía del empleo. Sin embargo, cuando el profesional de enfermería tiene una reducción en la capacidad de trabajo que lo aleja de la atención directa al paciente, existe un gran desafío para crear espacios de trabajo, y claras limitaciones en la adaptación de actividades, especialmente para la movilización y transporte de pacientes. Aquí se presentan las herramientas del Programa de Retorno al Trabajo y el rol del ergónomo en los casos de reincorporación al trabajo, las limitaciones del programa, los requisitos de la práctica profesional de enfermería y los desafíos de adaptarse al trabajo e incluir a las personas con restricciones laborales.
\end{abstract}

Palabras clave: Retorno al trabajo, enfermería, restricción laboral, ergonomía.

\begin{abstract}
This article accounts for the experience as an ergonomist in a Brazilian private hospital in training to support nursing professionals to return to work after prolonged periods of sick leave. The institutional development of a Return to Work Program involves a team seeking to guarantee support and employment. However, when the nursing professional reduces work capacity that takes him away from direct patient care, there is a great challenge to create workspaces and clear limitations in the adaptation of activities, especially for mobilization and patient transport. The paper presents the Return to Work Program tools and the role of the ergonomist in cases of return to work. Also, the program's limitations, the requirements of professional nursing practice and the challenges of adapting to work and including people with labor restrictions.
\end{abstract}

Keywords: Return to work, nursing, work restriction, ergonomics.

\footnotetext{
*Instituto de Docencia e Investigación Hospital Sírio Libanês. São Paulo, Brasil. Correo electrónico: angelica.gjuns@hsl.org.br. $\quad$ Orcid: https://orcid.org/oooo-0002-0869-5228. Autor de correspondencia.

**Instituto de Docencia e Investigación Hospital Sírio Libanês. São Paulo, Brasil. Correo electrónico: octavio.acoliveira@hsl.org.br. Orcid: https://orcid.org/o0oo-0002-8984-0430

****Instituto de Docencia e Investigación Hospital Śrio Libanês. São Paulo, Brasil. Correo electrónico: gabriela.mflorez@hsl.org.br. Orcid: https://orcid.org/oooo-0003-3902-5881
} 


\section{Introducción}

El Hospital Sirio Libanés (HSL) es un excelente hospital privado de la ciudad de Sao Paulo, referente internacional en salud, pionero en diferentes áreas como: inauguración de la primera UCI en el país e implementación, en el Servicio de Radioterapia, del primer acelerador latinoamericano de fotones y electrones lineales; inicio del primer programa de telemedicina en Brasil; realizar la primera telecirugía guiada por robot en el hemisferio sur; primer hospital de América Latina en realizar tomografía por emisión de positrones, entre otros. Conocido en Brasil por servir a políticos y celebridades, el hospital tiene un alto nivel de hospitalidad y satisfacción del cliente, con un enfoque en la experiencia del paciente, promoviendo la atención humanizada, con la calidez humana, el amor y la empatía como valores. En 2021, la unidad hospitalaria de São Paulo contaba con 7.400 trabajadores, de los cuales el equipo de enfermería contaba con unos 2.000 técnicos de enfermería y 900 enfermeras.

Por primera vez en su historia, solo en 2017 la institución contó con un ergónomo en su planta. La ergonomía comenzó a ser destacada, por el especialista de la institución, en las discusiones técnicas, como un recurso fundamental para la comprensión de las prácticas institucionales. Trabaja principalmente a demanda, en la valoración de las quejas de los trabajadores por malestar o sobrecarga, dificultades en la planificación del trabajo ante las limitaciones relacionadas con la enfermedad o variabilidad, y en el diseño de la obra, que puede ser una herramienta de prevención de enfermedades futuras y promoción de mejores condiciones de productividad. Se entendió que la ergonomía contribuye a mejorar la experiencia del trabajador y también la experiencia del paciente, convirtiéndose en aliado de varios programas institucionales.

Fue con esta premisa de mejorar e incluir la ergonomía como recurso para transformar las prácticas, es que el especialista se incorporó al equipo de salud ocupacional y participó en la creación del Programa de Retorno al Trabajo (PRT) en 2017. El programa tiene como objetivo apoyar al trabajador que, luego de un proceso de enfermedad, regresa al trabajo con una reducción en su capacidad de trabajo y la necesidad de un seguimiento para ajustar sus actividades laborales.

El equipo dedicado a discutir y tratar directamente las prácticas asistenciales en el retorno al trabajo está compuesto por 6 médicos ocupacionales, 3 enfermeras ocupacionales, 2 trabajadores sociales, 1 psiquiatra, 1 psicólogo y 1 terapeuta ocupacional ergónomo. Asimismo, 5 profesionales del sector de recursos humanos, dedicados a mediar decisiones y conflictos relacionados con el contrato laboral, los cuales se denominan socios comerciales, se incorporaron al proceso en 2018.

Inicialmente, el ergónomo contribuyó a la formación de profesionales de recursos humanos, médicos y enfermeras ocupacionales sobre la relación entre la evaluación de la funcionalidad y el análisis de actividades. Se buscó desarrollar una relación interdisciplinar con mayor alineación técnica y conceptual, llevando el análisis de la actividad laboral como 
algo transversal a las prácticas de todos los integrantes del equipo, para que el contenido del trabajo de los pacientes fuera más discutido por el equipo de salud.

La aplicabilidad del PRT en técnicos de enfermería fue elegida como el foco de discusión de este artículo, pertenecientes principalmente a los sectores de hospitalización y áreas críticas, ya que el equipo de salud ocupacional y los trabajadores de estas áreas enfrentan varios desafíos en el proceso de inclusión laboral, con limitaciones en sus funciones laborales, lo que es importante ser discutido y explorado para la reflexión sobre las prácticas.

\section{Materiales y métodos}

\section{Programa de regreso al trabajo (PRT)}

El programa se estructuró en torno a un material técnico: la "guía de un programa de prácticas integradas para apoyar la reincorporación al trabajo y la promoción de la retención laboral", desarrollado por el Instituto de Investigación en Salud y Seguridad Ocupacional de la Universidad de Laval, Quebec, Canadá, en 2014. Esto sirvió de base teórica para las discusiones y se adaptó a los flujos de la institución (ST-Arnaud \& Pelletier, 2014).

Luego nos dedicamos al desarrollo de flujos de servicios y la creación de formularios de evaluación que permitieran la formalización del proceso de análisis de los trabajadores, para el desarrollo de herramientas prácticas para los evaluadores profesionales y con lenguaje claro para los gerentes y trabajadores evaluados. La principal herramienta utilizada se denominó plan de trabajo compatible (PTC), que incluye los datos de la persona evaluada, la fecha de inicio de su ingreso al programa, la fecha propuesta de la próxima evaluación, y luego describió objetivamente las limitaciones funcionales en las actividades. que no se pueden realizar, con base en la Clasificación Internacional de Funcionalidad, y las adaptaciones necesarias en las actividades a desarrollar. Las evaluaciones funcionales consisten en un análisis del informe clínico y el historial ocupacional, además de evaluaciones clínicas de la estructura corporal. La construcción y validación de este documento incluye, como mínimo, al médico ocupacional, la enfermera ocupacional, el trabajador evaluado y su gerente inmediato.

\section{Evaluación de la funcionalidad según CIF}

Los términos y definiciones de la Clasificación Internacional de Funcionamiento, Discapacidad y Discapacidades (CIF) fueron adoptados como descriptores de las habilidades e incapacidades del sujeto (Buchalla, 2001), reemplazando la información sobre la enfermedad, antes del foco de las evaluaciones médicas. Su inserción facilita la comprensión de cómo la enfermedad se traduce en discapacidad, desde la perspectiva de la funcionalidad del sujeto, y qué actividades puede realizar un individuo que presenta cambios en su función y/o estructura corporal, así como su participación social. La discusión de la actividad, así como la funcionalidad e incapacidad de los individuos, se vuelve central. Asimismo, cobra relevancia la visión de que las funcionalidades están determinadas por el contexto, hay variabilidad intersubjetiva y también diferentes formas de actuar en el mundo, que difieren 
los enfoques para cada adaptación en el trabajo y hacen necesarios proyectos terapéuticos individuales (Organización Mundial de la Salud, 2013).

Valoración de la población de técnicos de enfermería atendidos

A partir de una base de datos de todos los PTC creados, fue posible realizar un análisis cuantitativo del perfil poblacional, distribución de los cuidados en el tiempo, tipo de limitación funcional y obtener un panorama de la representatividad de los técnicos de enfermería en la población atendida.

\section{Resultados y discusión}

La atención de los técnicos de enfermería en el Programa de Retorno al Trabajo

Entre los trabajadores atendidos, el $46 \%$ son técnicos de enfermería, cuyo desplazamiento a otra actividad es necesario, debido a la reducción del rendimiento físico o cognitivo que genera una enfermedad. Entre 2017 y 2021, el PRT brindó 582 servicios para adecuar las actividades laborales que culminaron en el PTC, de los cuales 268 estuvieron relacionados con técnicos de enfermería y, de estos, 133 trabajaron en el área de hospitalización de pacientes y unidades de cuidados intensivos.

A continuación, en la tabla 1, se observa la cuantificación realizada en registros de PTC dentro de esta población de 133 técnicos de enfermería (TE) en áreas de hospitalización de pacientes y unidades de cuidados intensivos. La distribución de PTC por año muestra una pequeña cantidad en 2017, ya que el período de servicio se restringió solo al mes de diciembre.

Tabla 1. Registros anuales de TE con PTC.

\begin{tabular}{|c|c|}
\hline Año & Cantidad TE con PTC \\
\hline 2017 & 10 \\
\hline 2018 & 29 \\
\hline 2019 & 57 \\
\hline 2020 & 37 \\
\hline
\end{tabular}

Valorando la principal limitación funcional descrita por los médicos del trabajo con respecto a los trabajadores evaluados, se agrupan en categorías que se muestran en la tabla 2. 
Tabla 2. Categorías de limitación funcional.

\begin{tabular}{|l|c|}
\hline Limitaciones funcionales & Cantidad TE \\
\hline Transportar de carga por encima de 5 kg & 32 \\
\hline Transportar de carga por encima de 10 kg & 22 \\
\hline Flexión o extensión de columna con o sin carga & 14 \\
\hline Realizar cuidados y comunicación directa con el paciente & 13 \\
\hline Funciones vinculadas a las extremidades superiores y los hombros & 11 \\
\hline Otros: post-operatorio específico & 10 \\
\hline Otros: fuera del estándar de evaluación funcional propuesto & 9 \\
\hline Caminar largas distancias & 4 \\
\hline Permanecer de pie o sentado durante un período prolongado & 4 \\
\hline Elevar carga mayor que 7 kg por encima de la línea del hombro & 4 \\
\hline Otras funciones relacionadas con las extremidades inferiores & 3 \\
\hline Empujar y tirar con requerimiento de fuerza (según límite individual) & 3 \\
\hline Levantar los brazos por encima de la línea de los hombros & 2 \\
\hline Manipulación manual de objetos de precisión & 1 \\
\hline Permanecer en cuclillas o de rodillas & 1 \\
\hline
\end{tabular}

Se observa que la restricción a la actividad de transporte manual de carga afecta al 40,6\% de los casos, la restricción a los movimientos de flexión o extensión de la columna vertebral es del 20,3\% de los casos, las limitaciones en la funcionalidad de los miembros superiores suman 8\%, y la limitación para el cuidado y la comunicación directa con el paciente, por problemas psicosociales, representan el 9,8\%; estas son las pérdidas funcionales más prevalentes.

Casi todos los trabajadores de enfermería con restricciones laborales no tienen ninguna indicación para mover a los pacientes (incluido el reposicionamiento en la cama y el transporte) o están contraindicados en relación directa con el paciente (para evitar condiciones de exposición al estrés y sobrecarga psicológica).

Del total de 133 TE, 78 de ellos, lo que equivale aproximadamente al 58,5\% de esta población, fueron asignados de manera permanente a las salas de preparación de medicamentos por sus gerentes. Los 55 trabajadores restantes, que comenzaron a tener una limitación laboral en relación a las actividades de asistencia directa, también fueron asignados temporalmente a salas de preparación de medicamentos. Pero, ¿por qué una respuesta tan homogénea a problemas tan diferentes?

El PRT tiene como objetivo permitir que los trabajadores actúen al máximo de su capacidad, contribuyendo a la organización y desarrollo personal. Sin embargo, los profesionales de la salud ocupacional identifican que cuando se trata de un técnico de enfermería que no puede soportar el peso del paciente durante el cuidado, está sujeto a estrés por conflicto con el paciente. La actividad de preparar medicamentos no requiere manipulación manual de cargas, elevación de miembros superiores, fuerza con brazos o piernas, flexión del tronco, ni los expone a la relación con los pacientes. Por ello, ha sido un lugar teóricamente "protegido" para trabajadores con limitaciones laborales. 


\section{Contribuciones de la ergonomía a las discusiones de casos}

El análisis del puesto de trabajo, previo a la pérdida de capacidad laboral, y del puesto de reincorporación, se realiza para demostrar si se ha producido o no un cambio significativo para el trabajador en aspectos físicos (espacio, manipulación de carga y postura), en aspectos organizativos (contenido de la actividad, ritmo de trabajo, división de tareas) y aspectos cognitivos (nivel de atención y vigilancia, recursos emocionales, toma de decisiones) (Silva \& Camarotto, 2016).

En el caso de cambiar la atención al paciente en las habitaciones, por la preparación de medicamentos, hay un factor muy importante a considerar: la atención al paciente y el conocimiento del técnico de enfermería. Algunos están interesados en cambiar el campo de acción, como ir al centro de materiales y esterilización o preparar medicamentos. Sin embargo, dado que la mayoría de ellos tienen conocimiento de la estructura organizacional y están sujetos a ella, se limitan a la sala de preparación de medicamentos y al desempeño de sus habilidades. Además, la enfermedad se convierte en un impedimento para el desarrollo profesional cuando hay una gran reducción en el alcance del trabajo. De esta forma, la enfermedad de los técnicos de enfermería afecta en gran medida su experiencia con el puesto, reduciendo su conciencia de pertenencia, autonomía profesional y seguridad con su empleo.

Con la visión de que debe haber un camino para el desarrollo de las personas y una organización del trabajo realizado para las personas, y no una estructura organizativa que las limite, el trabajo en ergonomía tiene como objetivo combatir esta lógica de que la única opción posible es cambiar a la sala de preparación de medicamentos. Sin embargo, el ergónomo mediante el diálogo con los gerentes de área sobre la planificación de actividades alternativas para estos trabajadores, recibió una retroalimentación de que es imposible mantener a uno de los trabajadores que no realiza todas las funciones en el marco de atención al paciente, ya que esto cambiaría significativamente la división de pacientes por profesional y sobrecargaría a otros profesionales. Tradicionalmente, la organización del trabajo en los sectores hospitalarios divide a los trabajadores entre la atención al paciente y la preparación de medicamentos. Estas actividades deben ser realizadas íntegramente por el profesional en cada línea de trabajo. Así, cuando se entiende que el profesional no puede formar parte del flujo de atención al paciente en la sala de hospitalización, se convierte automáticamente en preparador de medicamentos.

Además, la división del hospital entre gestión de exámenes y diagnóstico, hospitalización, cirugía, entre otros, se refleja en la contabilidad de los empleados por sector $\mathrm{y}$, en la lógica administrativa del sector de recursos humanos, no es posible ubicar a los trabajadores entre estos puestos de trabajo vacantes, lo que reduce las posibilidades de reubicación.

Ergónomo, médicos del trabajo, enfermeros, psicólogo y psiquiatra, recursos humanos y asistencia social se unieron para escuchar, discutir y transformar esta realidad. A través del análisis ergonómico es posible demostrar que los equipos para movilización de pacientes, las adaptaciones a la actividad y la mayor autonomía otorgada a los trabajadores pueden transformar el trabajo en un lugar más inclusivo. 
El ergónomo entonces comenzó a actuar para aportar mejoras a través de:

- Creación de rutas administrativas en recursos humanos para la reubicación del personal entre diferentes áreas del hospital, aumentando las posibilidades de desarrollo profesional y aprovechando el potencial de trabajo;

- Discutir con los gerentes el contenido y el potencial del trabajo con mayor importancia en vez de lo relacionado con la enfermedad o discapacidad;

- Dar a conocer la importancia de garantizar que permanezca en el trabajo y evitar más tiempo de inactividad, para un futuro más sostenible para la profesión;

- Actuar en la implementación de procesos de trabajo en los que el movimiento del paciente está principalmente mediado por la tecnología (ajuste de la cama, uso de accesorios y equipos como elevadores de pacientes), posibilitando que las personas con limitaciones al transporte manual de carga mantengan el cuidado del paciente.

Asimismo, para fortalecer el programa, también se identifican las siguientes necesidades:

- Mapeo más preciso de flujos e indicadores;

- Mejora del acercamiento de los gerentes con los trabajadores atendidos;

- Mayor dedicación entre los médicos ocupacionales para evaluar la funcionalidad basada en la Clasificación Internacional de Funcionamiento, Discapacidad y Salud (CIF);

- Ampliación del equipo para que sea posible contar con suficientes profesionales para, con mayor frecuencia, realizar el análisis de la actividad en base a principios ergonómicos y mediar, caso por caso, la decisión sobre qué adaptaciones y actividades pueden desarrollar estos trabajadores.

Además, la alta carga de trabajo de los profesionales de recursos humanos y salud ocupacional, que no tienen el PRT como práctica prioritaria, y la falta de un equipo dedicado y de profesionales capacitados para actuar con esto, genera frustración a los profesionales del equipo y a los trabajadores en el proceso de reincorporación al trabajo.

La valoración ergonómica de las actividades es fundamental para contribuir a la compatibilidad y diálogo entre las condiciones de salud de las personas y las exigencias de las actividades laborales (Simonelli, 2009). La no implicación del ergónomo en todas las evaluaciones solo se da porque esta condición requiere una amplia dedicación, que no se incluye en la carga de trabajo de dedicación de un solo profesional. Es también una premisa para el mejor desarrollo de este proyecto que se contrate a un terapeuta ocupacional para trabajar con ergonomía enfocado en el proceso de regreso al trabajo, capaz de dedicarse de lleno a los proyectos terapéuticos singulares desarrollados dentro del PRT. 


\section{Conclusiones}

La inclusión exitosa en nuevas actividades y la permanencia en el trabajo está relacionada con la implicación de varios profesionales, que deben orientar sus acciones en torno a un eje común y colectivamente predefinido. De lo contrario, los trabajadores se dirigen a trabajos que son incompatibles con su capacidad laboral, educación y calificaciones o quedan fuera del proceso de producción.

La experiencia de estos desafíos en equipo, aporta una experiencia valiosa. Se entiende que la composición del equipo multidisciplinar y el aporte de la ergonomía son factores fundamentales. Además, se tendrá paciencia, dedicación y se buscará el diálogo en discusiones con otras organizaciones. 


\section{Referencias}

Buchalla, C. M. (2001). CIF - Classificação Internacional de Funcionalidade, Incapacidade e Saúde. Universidade de São Paulo.

Organización Mundial de la Salud (2013). Cómo utilizar la CIF: Manual práctico para utilizar la Clasificación Internacional del Funcionamiento, la Discapacidad y la Salud (CIF). Versión preliminar para discusión. $\quad$ http://www.fsp.usp.br/cbcd/wpcontent/uploads/2015/11/Manual-Pra\%CC\%81tico-da-CIF.pdf

Silva, E. C., \& Camarotto, J. A. (2016). Contribuições da análise da atividade do trabalho no processo de retorno do trabalhador afastado. Revista De Terapia Ocupacional Da Universidade De São Paulo, 27(2), 131-137. https://doi.org/10.11606/issn.22386149.v27i2p131-137

Simonelli, A. P. (2009). Contribuições da análise da atividade e do modelo social para a inclusão no trabalho de pessoas com deficiência [tese doutorado, Universidade Federal de São Carlos]. Repositório Institucional da UFSCar. https://repositorio.ufscar.br/handle/ufscar/3331

St-Arnaud, L., \& Pelletier, M. (2014). Guide to an integrated practices program for supporting a return to work and promoting job retention. Institut de recherche Robert-Sauvé en santé et en sécurité du travail - Laval Uniersity. https://www.irsst.qc.ca/en/publicationstools/publication/i/100715/n/guide-return-to-work-absence-mental-health-rg-813 